\title{
On Signal Extraction and Non-Certainty-Equivalence in Optimal Monetary Policy Rules
}

\author{
Eric T. Swanson \\ Board of Governors of the Federal Reserve System \\ eswanson@frb.gov
}

\begin{abstract}
A standard result in the literature on monetary policy rules is that of certainty equivalence: given the expected values of all the state variables of the economy, policy should be set in a way that is independent of all higher moments of those variables. Some exceptions to this rule have been pointed out by Smets (1998), who restricts policy to respond to only a limited subset of state variables, and by Orphanides (1998), who restricts policy to respond to estimates of the state variables that are biased. In contrast, this paper studies unrestricted, fully optimal policy rules with optimal estimation of state variables. The rules in this framework exhibit certainty equivalence with respect to estimates of an unobserved, possibly complicated, state of the economy $X$, but are not certainty-equivalent when 1) a signal-extraction problem is involved in the estimation of $X$, and 2) the optimal rule is expressed as a reduced form that combines policymakers' estimation and policy-setting stages. In general, I show that it is optimal for policymakers to attenuate their reaction coefficient on a variable about which uncertainty has increased, while responding more aggressively to all other variables, about which uncertainty hasn't changed.
\end{abstract}

JEL Classification: E52

Version 0.4 .5

January 27, 2000

I thank Athanasios Orphanides, Glenn Rudebusch, David Small, and Volker Wieland for helpful discussions, comments, and suggestions. The views expressed in this paper, and all errors and omissions, should be regarded as those solely of the author, and are not necessarily those of the individuals listed above, the Federal Reserve System, or its Board of Governors. 


\section{Introduction}

Increased uncertainty about the current growth rate of productivity, potential output, and the natural rate of unemployment has led to questions about how monetary policy should be altered in the face of this uncertainty. The question is extremely important from a practical point of view (What should the Federal Reserve do today?), as well as being of particular theoretical interest (What are the various types of uncertainty faced by policymakers, and what effects should each of these have on optimal policy?)

A natural place to begin the investigation of these difficult questions is within the framework of monetary policy rules, such as those put forward by Taylor (1993). The advantage of these rules is that they are explicit, well-defined, and simple functions of variables within a completely specified economic model. Different types of uncertainty within the model can then be specified and their effects studied. The fact that Taylor-type rules have matched the historical behavior of the Federal Reserve for the past 15-20 years also lends support to their use as an analytical laboratory for the investigation of these issues.

A typical setup involves an economy that is linear in all of its variables, and policymakers who minimize an expected discounted sum of squared deviations of goal variables from their respective targets. The following backward-looking model serves as an illustrative example: ${ }^{1}$

$$
\begin{aligned}
\left(y_{t}-y^{*}\right) & =\varphi\left(y_{t-1}-y^{*}\right)-\alpha\left(r_{t}-r^{*}\right)+\varepsilon_{t} \\
\pi_{t} & =\pi_{t-1}+\beta\left(y_{t}-y^{*}\right)+\nu_{t}
\end{aligned}
$$

where $y_{t}, \pi_{t}$, and $r_{t}$ refer to output, inflation, and the real interest rate in period $t, y^{*}$ and $r^{*}$ denote the levels of potential output and the "natural" rate of interest consistent with long-run equilibrium, respectively, and $r_{t}$ is set by policymakers at the beginning of period $t$ based on information available through the end of period $t-1$. A typical

\footnotetext{
${ }^{1}$ I will also consider forward-looking models in the main body of the paper, below.
} 
specification of policymakers' preferences is:

$$
\min (1-\delta) E_{t} \sum_{s=t}^{\infty} \delta^{s-t}\left[\left(\pi_{s}-\pi^{*}\right)^{2}+\gamma\left(y_{s}-y^{*}\right)^{2}\right]
$$

a simple discounted sum of expected squared deviations of output from potential and inflation from its target, $\pi^{*}$, with weight $\gamma$ placed on the output gap. The case $\gamma=0$ corresponds to pure inflation targeting by policymakers, but does not necessarily prevent current or past values of the output gap from entering policymakers' optimal reaction function, since these variables may help forecast future values of inflation. Note that it is assumed in the model for simplicity that policymakers have control over the short-term real interest rate, $r_{t}$.

This is a simple, discrete-time dynamic programming problem with quadratic objective and linear constraints, the solution of which is well known (Sargent (1987)):

$$
r_{t}-r^{*}=a\left(y_{t-1}-y^{*}\right)+b\left(\pi_{t-1}-\pi^{*}\right)
$$

where $a$ and $b$ are constants, determined by the parameters of the model. The form of equation (3) and the values of $a$ and $b$ are the same no matter what the variances of $\varepsilon$ and $\nu$-policymakers behave in a certainty-equivalent fashion regardless of the variance associated with the laws of motion of the economy.

A major implication of this finding is that uncertainty about the level of potential output, $y^{*}$, should have no effect on the monetary policy rule. If at time $t$ we have $y^{*}=\hat{y}^{*}+\eta$, where $\eta$ is a mean-zero stochastic disturbance and $\hat{y}^{*} \equiv E_{t} y^{*}$, then the form of equations (1a) and (1b) is essentially unchanged, with $y^{*}$ replaced by $\hat{y}^{*}, \varepsilon_{t}$ replaced by $\tilde{\varepsilon}_{t} \equiv \varepsilon_{t}-(1-\varphi) \eta$, and $\nu_{t}$ replaced by $\tilde{\nu}_{t} \equiv \nu_{t}-\beta \eta$. Then, as before, the variances of $\tilde{\varepsilon}$ and $\tilde{\nu}$, and hence the variance of $\eta$, have no effect on the optimal policy rule-one simply replaces $y^{*}$ with $\hat{y}^{*}$ in equation (3) and acts as if this estimate $\hat{y}^{*}$ were known with certainty. This result is emphasized by Estrella and Mishkin (1998), and is standard in the literature on monetary policy rules.

To be sure, not all types of uncertainty are sterile when it comes to their effects on optimal policy. For example, if policymakers are unsure about the effects of their 
control variable $r_{t}$ on the economy, so that $\alpha$ is stochastic, the optimal choice of $r_{t}$ will depend on the degree of uncertainty underlying the parameter $\alpha$ (Brainard (1967), Sack (1998)). Here, the uncertainty is multiplicative with respect to the control variable, rather than being simply additive. ${ }^{2}$ In this paper, however, I abstract away from multiplicative uncertainty for a number of reasons. First, it is well known that certainty equivalence does not hold in the presence of multiplicative uncertainty. Second, the effects of multiplicative uncertainty on policymakers' optimal response function (3) are ambiguous in sign (when more than one coefficient is stochastic), and found by several authors to be quantitatively small in simulations (Estrella and Mishkin (1998), Rudebusch (1999a)). ${ }^{3}$ Finally, and most importantly, the uncertainty which policymakers appear to be most concerned with today is primarily additive in nature: Has there been a structural break in the growth rate of productivity, potential output, and the natural rate of unemployment? These are questions about the state variables of the economy themselves, not about the effects of choice variables on the economic state.

The main point of this paper is that additive uncertainty can have important effects on optimal policy, even within a linear-quadratic framework. Suppose, for example, that the state of the economy is $X_{t}$, an unobserved, possibly large vector. ${ }^{4}$ Suppose further that output and inflation are observable functions of this unobserved state of the economy $X_{t}$. Policymakers will behave in a certainty-equivalent fashion with respect to $\hat{X}_{t}$, the expected value of $X_{t}$, but will use observations of output and inflation to help infer what the value of $\hat{X}_{t}$ is. This inference stage of the problem, because it is one of signal extraction, is sensitive to the amount of uncertainty that is present in the observable variables of the system. In particular, as the noise in the data on the output gap, $y_{t}-y^{*}$, increases relative to the signal about $X_{t}$, it is optimal to decrease the coefficient on $y_{t}-y^{*}$ in the estimation of $\hat{X}_{t}$, and increase the coefficient on inflation in that estimation. Thus, the optimal policy, when expressed as a reduced-form function of output and inflation, rather

\footnotetext{
2 Note that uncertainty about $r^{*}, y_{t-1}$, and $\pi_{t-1}$ are also all additive with respect to the control variable $r_{t}$, and thus also have no effect on the optimal policy in (3).

${ }^{3}$ Sack (1998), however, finds larger effects of parameter uncertainty within a VAR framework.

${ }^{4}$ Some components of $X_{t}$ may be observed without altering the results.
} 
than $\hat{X}_{t}$, is not invariant to the degree of additive uncertainty in the former variables. It is important to note that both the rule itself and policymakers' estimate $\hat{X}_{t}$ are fully optimal in this framework - at every time $t$, policymakers' interest-rate choice and estimate of state variables are globally optimal, and cannot be improved upon in any way. This is one of the main respects in which the present paper differs from previous work in this area.

Previous studies of non-certainty-equivalence with additive uncertainty have followed either Smets (1998) or Orphanides (1998). Smets (1998) points out that certainty equivalence fails to hold when policymakers are constrained to respond to only a limited subset of the state variables of the system, so that the policy rule is a constrained optimum, rather than a global optimum. This restriction makes sense, in particular, when the size of the state space is large, as in Orphanides et al. (1999) and Drew and Hunt (1999), who work with the Federal Reserve Board's and Reserve Bank of New Zealand's macro models, respectively. ${ }^{5}$ Uncertainty about any of the variables in the policymakers' simple rule will then lead the optimal coefficients of the simple rule to change. ${ }^{6}$

Orphanides (1998) focuses on the fact that the data, such as the output gap and inflation rate, are observed only imperfectly in real time. By the certainty equivalence principle, policymakers' optimal response in this case is to formulate best estimates of the output gap, $E_{t}\left(y_{t}-y_{t}^{*}\right)$, and inflation, $E_{t} \pi_{t}$, and act as if these estimates were known with certainty. Orphanides and others (Aoki (1999), Rudebusch (1999a,b)) bring about non-certainty-equivalence in this framework by constraining policy to react to the actual real-time data, rather than to the best estimates above. This naturally raises the question as to why the actual real-time data are not (real-time) best estimates of the true values.

For example, if $y_{t}^{*}$ denotes the level of potential output, and $y_{t \mid t}^{*}$ the real-time esti-

\footnotetext{
${ }^{5}$ Actually, the RBNZ's macro model has an important nonlinearity in the relationship between inflation and unemployment. This implies that certainty equivalence will fail to hold even when policy is an optimal function of all state variables of the system. The FRB/US model, though nonlinear, is well-approximated by its linearization, and in fact it is the linearization that is used in Orphanides et al. (1999). Thus, certainty equivalence would hold in their paper if policy were allowed to be a function of all of the model's state variables, and the unobserved variables were estimated optimally.

${ }^{6}$ Uncertainty about any state variables not in the policymakers' simple rule will have no effect; this will be obvious from the discussion of the results in Section 2, below.
} 
mate of the level of potential output, one would normally expect that:

$$
y_{t}^{*}=y_{t \mid t}^{*}+\eta_{t}
$$

where $\eta_{t}$ is a mean-zero random variable. In this case, $y_{t \mid t}^{*}=E_{t} y_{t}^{*}$, so the real-time data $i s$ the real-time best estimate! The constraint that policymakers react only to the real-time data is then not really a constraint at all, and the certainty equivalence principle will hold.

It is only because Orphanides formulates the real-time data problem as one of signal extraction, with

$$
y_{t \mid t}^{*}=y_{t}^{*}+\eta_{t}
$$

where $y_{t}^{*}$ (the true value underlying the data, or signal) and $\eta_{t}$ (the noise) are orthogonal random variables, that the certainty equivalence principle is circumvented. Note that in $(4)^{\prime}$, the real-time data $y_{t \mid t}^{*}$ has the property that $y_{t \mid t}^{*} \neq E_{t} y_{t}^{*}$, so that it is no longer an unbiased estimate. When the problem is modified in this manner, an increase in the variance of $\eta_{t}$ now does have an effect on the best estimate $E_{t} y_{t}^{*}$-in particular, if $y_{t}^{*}$ and $\eta_{t}$ are normally distributed around zero, then $E_{t} y_{t}^{*}=\left(\sigma_{y^{*}}^{2} /\left(\sigma_{y^{*}}^{2}+\sigma_{\eta}^{2}\right)\right) y_{t \mid t}^{*}$ in the univariate case. Although the optimal policy is still a certainty-equivalent function of policymakers' best estimate $E_{t} y_{t}^{*}$, expressing the policy as a function of the real-time $y_{t \mid t}^{*}$ now leads to effects of additive uncertainty $\eta_{t}$ on the coefficients in the optimal rule. It is in this respect that Orphanides (1998) finds an exception to the certainty equivalence principle.

It should be clear from the above analysis that the use of real-time data per se has nothing to do with the exception to certainty equivalence demonstrated in Orphanides (1998). Instead, it is the introduction of a signal extraction problem into the policymakers' inference step that drives the result. The general implications of formulating policymakers' inference problem in this way is the domain of the present paper. In addition, the noncertainty-equivalent policies that are emphasized in Orphanides (1998) and Rudebusch (1999a,b) are not fully optimal - they can be improved upon by making use of additional lags of the data, for example. Thus, it is difficult in those papers to distinguish between the effects of uncertainty in the inference step of the problem versus the effects of uncertainty that arise from using a substantially constrained policy response function. In the present 
paper, all policy rules and all estimation will be fully optimal. Again, this is one of the main points of departure from previous work.

Section two of the paper develops the relationship between signal extraction and noncertainty-equivalence in a simple descriptive model of the economy, under both naive and rational expectations. Section three extends these results to the general linear-quadraticGaussian framework and proves the coefficient attenuation result for the general case. Section four extends the basic model to allow for dynamic evolution of uncertainty, and proves that the results of section two are robust to this extension. Section five discusses the results and concludes.

\section{The Basic Model}

Policymakers have preferences over inflation and output of the form:

$$
\min (1-\delta) E_{t} \sum_{s=t}^{\infty} \delta^{s-t}\left[\left(\pi_{s}-\pi^{*}\right)^{2}+\gamma\left(y_{s}-y^{*}\right)^{2}\right]
$$

where $\pi^{*}$ denotes policymakers' long-run target for the inflation rate and $y^{*}$ denotes the level of "potential" output consistent with long-run equilibrium. The case $\gamma=0$ corresponds to pure inflation targeting, but does not necessarily preclude current or past values of the output gap from entering policymakers' optimal reaction function, since they may help forecast inflation.

For the purposes of this section, the unobserved "signal" variable $X_{t}$ will be taken to be a scalar. The interpretation in this case is that the true underlying state of the economy is scalar, or alternatively that $X_{t}$ is an index of inflationary pressures, this index itself being an amalgam of a great many variables underlying the economy. In either case, $X_{t}$ is assumed to evolve according to:

$$
X_{t}=\varphi X_{t-1}-\alpha\left(r_{t}-r^{*}\right)+\varepsilon_{t}
$$

while the output gap and inflation are observable functions of this unobserved state:

$$
\begin{aligned}
\left(y_{t}-y^{*}\right) & =X_{t}+\eta_{t} \\
\pi_{t} & =\pi_{t}^{e}+\beta X_{t}+\nu_{t}
\end{aligned}
$$


Here $r^{*}$ denotes the "natural" rate of interest, consistent with long-run equilibrium, and $\alpha, \beta$, and $\varphi$ are known positive parameters with $\varphi<1$. The stochastic disturbances $\varepsilon_{t}, \eta_{t}$, and $\nu_{t}$ are independent of each other, over time, of current and prior values of $X, y$, and $\pi$, and are normally distributed with constant variances $\sigma_{\varepsilon}^{2}, \sigma_{\eta}^{2}$, and $\sigma_{\nu}^{2}$, respectively. The variable $X_{t}$ (and its past values) are never observed by policymakers and must be inferred from previous observations of output and inflation. Equations (6b) and (6c) represent the signal extraction aspect of the problem, with $\eta_{t}$ and $\nu_{t}$ denoting noise disturbances that are orthogonal to the underlying signal $X_{t}$.

One may assume that $y^{*}$ is observed with certainty, or alternatively that it is stochastic $\left(\pi^{*}\right.$ and $r^{*}$ are presumed to be known with certainty, although in principle these restrictions, too, could be dropped). Letting $y^{*}=\hat{y}^{*}+\zeta$, where $\zeta$ is a random variable, one can rewrite equation $(6 \mathrm{~b})$ as:

$$
\left(y_{t}-\hat{y}^{*}\right)=X_{t}+\tilde{\eta}_{t}
$$

where $\tilde{\eta}_{t} \equiv \eta_{t}+\zeta$ is orthogonal to $X_{t}{ }^{7}$ From the point of view of policymakers' decision at time $t$, this is equivalent to simply increasing the variance of $\eta_{t}$ in equation (6b), so the analysis is simplified, without loss of generality, by restricting attention to that equation, and studying the effects of an increase in $\sigma_{\eta}^{2}{ }^{8}$

Equation (6c) also incorporates economic agents' prior expectation of the inflation rate, $\pi_{t}^{e}$, which is known and fixed at the beginning of period $t$. In principle, this could be a rational expectation $\left(\pi_{t}^{e} \equiv E_{t} \pi_{t}\right.$, where $E_{t}$ denotes the expectation at the beginning of period $t$, before shocks are realized), a naive adaptive expectation $\left(\pi_{t}^{e} \equiv \pi_{t-1}\right)$, or a forecast derived by some other means. In practice, the special linear-quadratic structure of the problem is lost unless $\pi_{t}^{e}$ is either a rational expectation or a fixed linear combination

\footnotetext{
${ }^{7}$ Orthogonality requires that the error $\zeta$ be orthogonal to $X_{t}$. This is the case, for example, when the uncertainty surrounding $y^{*}$ is due to a regime change that has been known to occur in the previous period, so that $y^{*}$, which was previously known with certainty, is suddenly uncertain. The more general case, where $\zeta$ may be correlated with $X_{t}$, is not difficult, and is solved in Sections 3 and 4 , below. The results there are essentially identical to those presented here.

${ }^{8}$ There is one complication in that uncertainty about $y^{*}$ diminishes over time (in the absence of new shocks to $y^{*}$ ) as policymakers learn from observations of $y$ and $\pi$. This poses no problem, however, because the separation of estimation and control in this linear-quadratic framework implies that this learning process (which takes place via Kalman filtering) has no effect on the optimal policy (see, for example, Bertsekas (1987), p. 292).
} 
of finitely many lags of observable variables; thus, that assumption will be maintained here. In addition, for the purposes of this section, it will be assumed that $\pi_{t}^{e}$ is either rational or purely naive $\left(\pi_{t}^{e} \equiv \pi_{t-1}\right)$, as this keeps the number of state variables in the model to a minimum. ${ }^{9}$

The timing of policymakers' observations and actions is as follows. At the beginning of period $t$, policymakers update their beliefs about $X_{t-1}$ based on observations of $y_{t-1}$, $\pi_{t-1}$, and the earlier choice of $r_{t-1}$. Based on these updated beliefs, policymakers then choose a value of $r_{t}$ that minimizes the expected loss function (5). Shocks to the economy $\left(\varepsilon_{t}, \eta_{t}\right.$, and $\left.\nu_{t}\right)$ are then realized and the values $y_{t}$ and $\pi_{t}$ observed. Thus, policymakers' information set at time $t$ is:

$$
\mathcal{I}_{t} \equiv\left\{\alpha, \beta, \delta, \varphi, \sigma_{\varepsilon}^{2}, \sigma_{\eta}^{2}, \sigma_{\nu}^{2}, E_{0} X_{0}, \operatorname{Var}_{0} X_{0}, \pi^{*}, r^{*}, y^{*}, \pi_{t}^{e}, \pi_{s}^{e}, \pi_{s}, r_{s}, y_{s} \mid s<t\right\}
$$

where $E_{0} X_{0}$ and $\operatorname{Var}_{0} X_{0}$ denote the mean and variance of policymakers' prior (time 0 ) distribution on $X_{0}$, which is assumed to be normal.

Policymakers update beliefs about $X_{t-1}$ via Kalman filtering. Because $\left(\varepsilon_{t}, \eta_{t}, \nu_{t}\right)$ is multivariate normally distributed, this is the optimal inference procedure (minimizing the mean-squared error of the estimate), and is equivalent to Bayesian updating. ${ }^{10}$

The optimal solution to policymakers' problem (5), given the structure of the economy (6) and information set (7), is:

$$
r_{t}=r^{*}+a E_{t} X_{t-1}+b\left(\pi_{t}^{e}-\pi^{*}\right)
$$

where $a$ and $b$ are positive constants determined by the parameters of the system. ${ }^{11}$ Note that $a$ and $b$ are completely invariant to $\sigma_{\varepsilon}^{2}, \sigma_{\eta}^{2}, \sigma_{\nu}^{2}$, and $\operatorname{Var}_{t} X_{t-1}$ (policymakers' (time $t$ )

\footnotetext{
${ }^{9}$ It is not difficult to show (by introducing a coefficient on $\pi_{t}^{e}$ in (6c) and letting it tend to one) that when $\pi_{t}^{e}$ is rational, policymakers can set $E_{t} \pi_{t}=\pi^{*}$ and $E_{t} y_{t}=y^{*}$ in every period, and thus $\pi_{t}^{e}=\pi^{*}$ in every period. See Appendix A. Also note that the exact process by which agents' expectations are formed has no effect on policymakers' period $t$ estimate of $X_{t}$. It is only because of the control aspects of the problem that assumptions about agents' expectation formation are required.

${ }^{10}$ Without any assumptions regarding the distribution of $\left(\varepsilon_{t}, \eta_{t}, \nu_{t}\right)$, Kalman filtering is still the optimal linear inference procedure. However, the assumption of normality, or other more complicated constraints on policymakers' estimation and control, are required in order to generate separability of estimation and control in the policymakers' problem.

${ }^{11}$ This is most easily seen by solving the policymakers' problem explicitly. See Appendix A.
} 
prior variance on $X_{t-1}$, derived recursively from $\operatorname{Var}_{0} X_{0}$ by the Kalman filtering algorithm). In this respect, the linear-quadratic problem with signal extraction continues to display certainty equivalence.

In forming the optimal estimate $E_{t} X_{t-1}$, however, policymakers $d o$ respond to the amount of uncertainty in the problem. Their prior (time $t-1$, i.e. before values of $y_{t-1}$ and $\pi_{t-1}$ are observed) distribution on $\left(X_{t-1}, y_{t-1}-y^{*}, \pi_{t-1}-\pi_{t-1}^{e}\right)$ is given by:

$$
\begin{aligned}
& \left(X_{t-1}, y_{t-1}-y^{*}, \pi_{t-1}-\pi_{t-1}^{e}\right) \sim \\
& \quad N\left(\left[\begin{array}{c}
\varphi E_{t-1} X_{t-2}-\alpha\left(r_{t-1}-r^{*}\right) \\
\varphi E_{t-1} X_{t-2}-\alpha\left(r_{t-1}-r^{*}\right) \\
\beta \varphi E_{t-1} X_{t-2}-\beta \alpha\left(r_{t-1}-r^{*}\right)
\end{array}\right],\left[\begin{array}{ccc}
\sigma_{x}^{2} & \sigma_{x}^{2} & \beta \sigma_{x}^{2} \\
\cdot & \sigma_{x}^{2}+\sigma_{\eta}^{2} & \beta \sigma_{x}^{2} \\
\cdot & \cdot & \beta^{2} \sigma_{x}^{2}+\sigma_{\nu}^{2}
\end{array}\right]\right)
\end{aligned}
$$

where I have let $\sigma_{x}^{2}$ denote $\operatorname{Var}_{t-1} X_{t-1}$, policymakers' prior (time $t-1$ ) variance on $X_{t-1}$. Their posterior distribution on $X_{t-1}$ (after observing $y_{t-1}$ and $\pi_{t-1}$ ) then has mean:

$$
\begin{aligned}
E_{t} X_{t-1} & =\varphi E_{t-1} X_{t-2}-\alpha\left(r_{t-1}-r^{*}\right) \\
& +\frac{\sigma_{x}^{2}\left(\beta^{2} \sigma_{x}^{2}+\sigma_{\nu}^{2}\right)-\left(\beta \sigma_{x}^{2}\right)^{2}}{\Delta}\left(\left(y_{t-1}-y^{*}\right)-\varphi E_{t-1} X_{t-2}+\alpha\left(r_{t-1}-r^{*}\right)\right) \\
& +\frac{-\beta \sigma_{x}^{4}+\beta \sigma_{x}^{2}\left(\sigma_{x}^{2}+\sigma_{\eta}^{2}\right)}{\Delta}\left(\left(\pi_{t-1}-\pi_{t-1}^{e}\right)-\beta \varphi E_{t-1} X_{t-2}+\beta \alpha\left(r_{t-1}-r^{*}\right)\right)
\end{aligned}
$$

where $\Delta \equiv\left(\sigma_{x}^{2}+\sigma_{\eta}^{2}\right)\left(\beta^{2} \sigma_{x}^{2}+\sigma_{\nu}^{2}\right)-\left(\beta \sigma_{x}^{2}\right)^{2}=\sigma_{x}^{2} \sigma_{\nu}^{2}+\beta^{2} \sigma_{x}^{2} \sigma_{\eta}^{2}+\sigma_{\eta}^{2} \sigma_{\nu}^{2}$. Equation (9) is analogous to the simpler formula for signal extraction with one observable variable, $E_{t} X_{t-1}=E_{t-1} X_{t-1}+\left(\sigma_{x}^{2} /\left(\sigma_{x}^{2}+\sigma_{\eta}^{2}\right)\right)\left(\left(y_{t-1}-y^{*}\right)-E_{t-1}\left(y_{t-1}-y^{*}\right)\right)$, with additional terms in the coefficients that take into account the covariance between output and inflation. ${ }^{12}$

Grouping terms in (9) yields:

$$
E_{t} X_{t-1}=\frac{\sigma_{\eta}^{2} \sigma_{\nu}^{2}}{\Delta}\left(\varphi E_{t-1} X_{t-2}-\alpha\left(r_{t-1}-r^{*}\right)\right)
$$

${ }^{12}$ Given the normality assumption, the formula for the best predictor $E_{t} X_{t-1}$ is the theoretical regression/projection:

$$
E_{t} X_{t-1}=E_{t-1} X_{t-1}+\operatorname{Cov}_{t-1}\left(X_{t-1}, Z_{t-1}\right)\left(\operatorname{Var}_{t-1} Z_{t-1}\right)^{-1}\left(Z_{t-1}-E_{t-1} Z_{t-1}\right)
$$

where $Z_{t-1} \equiv\left[\begin{array}{c}y_{t-1}-y^{*} \\ \pi_{t-1}-\pi_{t-1}^{e}\end{array}\right]$. This yields:

$$
E_{t} X_{t-1}=E_{t-1} X_{t-1}+\left[\begin{array}{ll}
\sigma_{x}^{2} & \beta \sigma_{x}^{2}
\end{array}\right]\left[\begin{array}{cc}
\sigma_{x}^{2}+\sigma_{\eta}^{2} & \beta \sigma_{x}^{2} \\
\cdot & \beta^{2} \sigma_{x}^{2}+\sigma_{\nu}^{2}
\end{array}\right]^{-1}\left(Z_{t-1}-E_{t-1} Z_{t-1}\right)
$$

which is the expression given in (9). 


$$
\begin{aligned}
& +\frac{\sigma_{x}^{2} \sigma_{\nu}^{2}}{\Delta}\left(y_{t-1}-y^{*}\right) \\
& +\frac{\beta \sigma_{x}^{2} \sigma_{\eta}^{2}}{\Delta}\left(\pi_{t-1}-\pi_{t-1}^{e}\right)
\end{aligned}
$$

where the $E_{t-1} X_{t-2}$ term can be cascaded backward and expressed as a function of lagged observations of $y, \pi$, and $r$ to the point where the original distribution on $X_{0}$ is negligible, as it is multiplied by a large power of $\varphi$.

Note that, even though policymakers' optimal reaction function (8) is certaintyequivalent in terms of the estimate $E_{t} X_{t-1}$, when expressed as a function of present and past observable variables $(y, \pi$, and $r)$, certainty equivalence in the reduced form no longer holds. The variances of the additive disturbance terms enter into the coefficients of (10).

For example, consider the effects of an increase in $\sigma_{\eta}^{2}$ on the coefficients in equation (10). ${ }^{13}$ As $\sigma_{\eta}^{2}$ increases, the quantity $\Delta$ increases, but less than proportionately. This implies that the coefficient on $y_{t-1}-y^{*}$ in (10) decreases in magnitude, so that policymakers place less weight on the observation of the uncertain output gap in forming their inference about the underlying state of the economy. Thus, we have an example of coefficient attenuation on the noisy or uncertain variable. In addition, the coefficients on each of the other variables in equation (10) increase in absolute value: policymakers place more weight on those variables about which they are relatively more certain. In this sense, policymakers become "less proactive and more reactive," responding less forcefully to the current value of the output gap, and more forcefully to past observations of output and inflation, and the current inflation rate, because these variables provide more reliable information about the current state of the economy and future values of policymakers' goal variables, inflation and output. ${ }^{14}$

\footnotetext{
${ }^{13}$ As noted above in footnotes 7 and 8 , this can be thought of as corresponding to a structural break in $y^{*}$ which has been known to occur in the previous period. This causes $\sigma_{\eta}^{2}$ to rise for the current period but not for previous periods, and hence leaves $\sigma_{x}^{2}$ unaffected.

14 This "less proactive" result could be emphasized further by setting up the main model with an additional lag in equation (6c), so that:

$$
\pi_{t}=\pi_{t}^{e}+\beta X_{t-1}+\nu_{t}
$$

This approach, though more interesting, was not pursued in this section, because it makes the signal extraction problem a function of updates of two lags of the variable $X_{t}$ instead of only one.
} 
These conclusions are not idiosyncratic to an increase in the variance of the additive disturbance $\eta_{t}$. For example, an increase in $\sigma_{\nu}^{2}$, instead of $\sigma_{\eta}^{2}$, leads to a decrease in the coefficient on $\left(\pi_{t-1}-\pi_{t-1}^{e}\right)$ in equation (10), and an increase in the coefficients on the other variables in that equation. Exactly analogous results (lower coefficient on the noisy variable, higher coefficients on the others) obtain in response to an increase in $\sigma_{x}^{2}$, the policymakers' prior variance on $X_{t-1}$. Thus, the result described above is robust, and derives not from any special assumptions surrounding the model, but rather from the general principle that in linear regression, or statistical projection, less weight is given to observations that have higher variance.

Obviously, as with linear regression and statistical projection, the covariances between the different variables matters for the coefficients in (10). So far, I have abstracted away from this problem by assuming that the disturbances are orthogonal to each other, and to policymakers' prior for the variable $X_{t-1}$, but I show below for the general case, with arbitrary covariances, that the basic coefficient attenuation result still holds: an increase in the uncertainty surrounding a given variable causes policymakers to assign less weight to that variable in forming their best estimate of the underlying state of the economy, $E_{t} X_{t-1}$. Moreover, the amplification of the coefficients on inflation and its lags in (10) is also quite robust, and holds for models more general than that of the present section. For example, section 4 proves this result for the case where increased uncertainty about potential output extends backward any number of periods, is correlated across time, and is correlated with policymakers' priors about the unobserved state of the economy $X_{t-1}$, in a manner which is consistent with policymakers learning about potential output over time.

Finally, the direction that the coefficients in equation (10) move can be ambiguous when one increases the variance of more than one variable at a time. For example, increas$\operatorname{ing} \sigma_{\eta}^{2}$ and $\sigma_{x}^{2}$ simultaneously causes the coefficient on the unaffected variable $\left(\pi_{t-1}-\pi_{t-1}^{e}\right)$ to increase unambiguously, but the effect on the other two coefficients is not clear. ${ }^{15}$ I will

\footnotetext{
${ }^{15}$ Although if $\sigma_{\eta}^{2}$ increases by more than $\sigma_{x}^{2}$, it is easy to show that the coefficient on $\left(y_{t-1}-y^{*}\right)$ must decrease in magnitude, while if $\sigma_{x}^{2}$ increases by more than $\sigma_{\eta}^{2}$, then the coefficient on $E_{t-1} X_{t-2}$ must decrease in magnitude.
} 
return to this issue in section 4 , below, but will remove the ambiguity by making use of the explicit relationships between $\sigma_{x}^{2}$ and past values of $\sigma_{\eta}^{2}$ that arise from Kalman filtering.

\subsection{Signal Extraction vs. Imperfect Observation of State Variables}

It is important to note that the above results hinge crucially on setting up model (6) as one involving signal extraction, rather than one simply involving imperfect observation of state variables. For example, replacing (6b) with:

$$
X_{t}=\left(y_{t}-y^{*}\right)+\eta_{t}
$$

or, with an uncertain potential output:

$$
X_{t}=\left(y_{t}-\hat{y}^{*}\right)+\tilde{\eta}_{t}
$$

where $y^{*} \equiv \hat{y}^{*}+\zeta$ and $\tilde{\eta}_{t} \equiv \eta_{t}-\zeta$, the non-certainty-equivalence results described above are completely eliminated. In both $(6 \mathrm{~b})^{\dagger}$ and $(6 \mathrm{~b})^{\ddagger}$, the policymaker's optimal estimate of the underlying state of the economy, $E_{t} X_{t-1}$, is simply $\left(y_{t-1}-y^{*}\right)$ in the first case, and $\left(y_{t-1}-\hat{y}^{*}\right)$ in the second. This estimate is certainty-equivalent, and plugged into the certainty-equivalent structural response in (8), the reduced-form policy response retains the certainty-equivalence property.

This implies that it is not noisy data per se that justifies caution on the part of policymakers in Orphanides (1998) and Rudebusch (1999a). If the real-time data are unbiased forecasts of the true values, analogous to $(6 \mathrm{~b})^{\dagger}$ or $(6 \mathrm{~b})^{\ddagger}$, then certainty equivalence holds, and the amount of uncertainty surrounding these real-time estimates is completely irrelevant. Certainty equivalence only fails to hold in their framework if the real-time data are realizations of true values plus noise (and thus are biased forecasts of the true values), so that estimation of the true values involves a signal extraction problem as in (6b).

This naturally raises the question as to whether the real-time data are better modeled as rational forecasts or as noise-contaminated realizations. Orphanides $(1998,1999)$ 
presents figures demonstrating that the output gap, in particular, has been badly mismeasured by policymakers in real time. However, both the size of these errors and their serial correlation were not evident until several years after the fact, so it is not clear that these real-time estimates of the output gap were not rational forecasts at the time. To take the position that policymakers were deliberately irrational in their real-time estimates seems unwarranted without a more rigorous analysis of policymakers' forecasts to support this point of view.

Rigorous analysis of the performance of real-time data and official forecasts has been undertaken by a number of authors, albeit with data that is more readily observable than the output gap, such as real GDP and inflation. Mankiw and Shapiro (1986) analyze whether the real-time real GNP data produced by the BEA is better modeled as a rational forecast or as a realization with noise of the "true" (i.e., final) value. They find that the real-time data appear to be unbiased and efficient rational forecasts. McNees (1995) looks at the official forecasts of real GNP/GDP and inflation put out by the CBO and Federal Reserve System (as presented in "Humphrey-Hawkins" reports) and finds that they perform at least as well, if not better, than private-sector forecasts in terms of meansquared error. Internal studies at the Federal Reserve Board have also found that the Board's internal "Greenbook" forecasts of real GNP/GDP and inflation are unbiased and efficient (Reifschneider (199?)). This is further supported by Romer and Romer (2000), who find that not only are the Federal Reserve Board's forecasts of output and inflation unbiased and efficient, they completely dominate private sector forecasts, in the sense that the private sector forecast should be thrown out entirely if the Board's forecasts were to be made public.

These results might at first seem to contradict Rudebusch's (1999a) finding of a significant, irrational "noise" component in the real-time inflation data (as measured by either the GNP/GDP deflator or fixed-weight price index). However, as in Orphanides (1998, 1999), Rudebusch's "final" data is from the perspective of the late 1990's, and thus includes definitional revisions to GNP and changes in base year. In contrast, all of the papers cited above take particular care to evaluate the performance of the forecasts with 
respect to a final measure of the statistic on a definitionally consistent basis. As McNees (1995) points out, "It does not seem reasonable to hold economic forecasters responsible for anticipating such changes in the social accounting framework" (p. 15). Thus, it is possible that much of the "noise" that is found by Rudebusch (1999a) is related to definitional changes in the data rather than deviations from rationality in the forecasts.

The case for biased real-time data is thus somewhat uncompelling from an empirical as well as a theoretical standpoint. This implies that models incorporating only real-time data uncertainty should be certainty-equivalent. However, framing policymakers' inference problem about the state of the economy as one of signal extraction more generally, as is done in this paper, is still quite plausible. The interpretation of policymakers' estimation process in this case is one of an unobserved, possibly complicated state of the economy that must be inferred using (possibly a large number of) observable economic indicators. For example, Chairman Greenspan is notorious for looking at a wide variety of economic statistics in an attempt to infer the current state of the economy and its future course. When policymakers face a signal extraction problem of this kind, as in model (6) above and the general framework below, a strong case for policymaker caution in the face of uncertainty can still be made.

\subsection{Coefficient Attenuation, Simple Rules, and Robust Control}

The implications of this paper contrast in an interesting way with those from the literature on "simple rules" and "robust control." In particular, the signal extraction framework of this paper implies that an increase in uncertainty surrounding a given indicator should be met with an attenuation in policymakers' response coefficient to that variable. At the same time, policymakers' response coefficients to other economic indicators, about which uncertainty hasn't changed, should be increased in magnitude. ${ }^{16}$

The literature on "simple rules," in contrast, generally finds that policymakers

\footnotetext{
${ }^{16}$ In the completely general setup of the model (Section 3), only the coefficient attenuation result on the uncertain variable holds unambiguously. However, both in the simple framework (6) above and its generalization to uncertainty extending back any number of periods below (section 4), amplification of coefficients on the other variables, about which uncertainty has not increased, holds generally.
} 
should attenuate their response coefficients on all variables in policymakers' reaction function, even if the increase in uncertainty surrounds only a single variable. ${ }^{17}$ This literature, typified by Smets (1998), considers optimal policy within a class of rules that react only to a limited subset of state variables of the model. These simple rules, because they are not the global optimum of policymakers' linear-quadratic problem, will not in general possess the certainty-equivalence property. Thus, observation error on a variable, even of the type in $(6)^{\dagger}$ and $(6)^{\ddagger}$, will typically lead to non-certainty-equivalent behavior with respect to these constrained-optimal rules.

For example, Smets (1998) restricts policymakers to rules involving only one lag of output, the four-quarter average inflation rate, and one lag of the interest rate as arguments, and finds that the optimal coefficients on all of these variables are attenuated by an increase in uncertainty surrounding the output gap. Orphanides et al. (1999), investigating a similarly-constrained class of rules within the Federal Reserve Board's FRB/US model, also find that attenuating the coefficients on all variables in the rule is the best response to increased uncertainty surrounding the output gap. ${ }^{18}$ The reason that these findings differ from those of the present paper can be explained as follows. The optimal rule in all of these models is a function of multiple lags of the output gap, inflation, and interest rates (and many other variables in the FRB/US model). To the extent that these variables are omitted, those that enter the simple rule serve partially as proxies for the variables that have been excluded. If the four-quarter average inflation rate enters negatively into an estimation equation for some of these other terms (such as past values of the interest rate), then the desired amplification in coefficients on current and past inflation

\footnotetext{
17 The only exception, to my knowledge, is Drew and Hunt (1999), who find that policymakers should increase their response coefficients on all variables in response to output gap uncertainty. Their finding is idiosyncratic for several reasons. First, the RBNZ's macro model is significantly nonlinear, so that certainty equivalence would fail to hold even with a fully optimal rule. Second, policymakers' estimate of potential output is irrational, using an HP-filter that yields estimates that are significantly correlated with the business cycle. Finally, policymakers are not permitted to ever revise their estimate of potential output in the future; they thus have an incentive to react strongly to deviations of inflation from target and output from estimated potential so that the economy does not swing too far away from these values.

18 Orphanides et al. do find that under some circumstances an amplification in the coefficient on the inflation rate is optimal. However, this only occurs when they increase the relative weight on the output gap in policymakers' loss function to high levels ( 0.75 or above).
} 
that I find will be offset by the desired amplification in the coefficients on these additional lagged output, inflation, and interest rate terms, making the overall effect on the inflation coefficient ambiguous. ${ }^{19}$

Finally, the "robust control" literature arrives at just the opposite prescription: policymakers ought to respond more aggressively to every variable in their reaction function when faced with model uncertainty. This literature, typified by Onatski and Stock (1998), chooses coefficients on a policy rule to minimize the maximum loss over all possible values for a given parameter within a given range; thus, the policymaker is guaranteed not to make mistakes that are extremely costly. This approach is clearly very different from the typical maximization of expected value approach I have taken here, so it is not surprising that the results differ. Intuitively, their findings are driven by the fact that a bad draw on the effectiveness of the policy tool (a multiplicative parameter) can result in very large losses if the rule's responsiveness is not sufficiently great. However, it is not clear that an increase in additive uncertainty about potential output would lead to the same conclusions.

\section{Signal Extraction in the General LQG Framework}

In this section, I solve the policymakers' signal extraction and optimization problem for the general linear-quadratic-Gaussian framework, and provide the relevant proofs.

As before, I denote the underlying state of the economy by $X_{t}$, which may now be a vector. $X_{t}$ evolves according to a linear function of one lag of itself and a vector of policy instruments $r_{t}$. Thus,

$$
X_{t}=A X_{t-1}+B r_{t}+\varepsilon_{t}
$$

where $A$ and $B$ are known matrices of the approrpriate dimensions. Any constants can be incorporated by defining one component of $X_{t}$ to be a vector of ones. I denote the

\footnotetext{
${ }^{19}$ There are some other minor differences at work as well. For example, Smets evaluates the effects of increases in the variance of the output gap that go back into the infinite past, while the experiment considered in this section has been more closely related to a change in regime or structural break at a given time $t$. However, I show in section 4, below, that the results of this paper continue to hold when uncertainty about the output gap extends backward any number of periods. Also, Smets and Orphanides et al. constrain their simple policy rules to be functions of inflation deviations from target (the four-quarter moving average of $\left.\left(\pi_{t-1}-\pi^{*}\right)\right)$ rather than the inflation surprise $\left(\pi_{t-1}-\pi_{t-1}^{e}\right)$ which would be closer to the prescriptions of the optimal inference procedure in (10).
} 
observable variables of the system by $Z_{t}$. These may be a subset of the variables in $X_{t}$, noisy realizations of a linear function of variables in $X_{t}$, or some combination of the two. Thus,

$$
Z_{t}=C X_{t}+\eta_{t}
$$

where $C$ is a known matrix of appropriate dimension, with every observable relationship among the elements of $X_{t}$ corresponding to a row in (12). The noise vector $\eta_{t}$ may have some components that are always zero, corresponding to elements of $X_{t}$ that are actually observed. Other components of $X_{t}$, that are not directly observed, must be inferred from observations of $Z_{t} \cdot{ }^{20}$ Note that (12) has been set up as a signal extraction problem rather than one of imperfect observation (in which case $X_{t}$ would be a function of the observable variables $Z_{t}$ plus disturbance terms). The latter would exhibit certainty equivalence; the former does not (with respect to the observable variables).

The stochastic disturbances $\varepsilon_{t}$ and $\eta_{t}$ are assumed to be independent of each other, over time, of current and past values of $r, X$, and $Z$, and are (multivariate) normally distributed with constant variance-covariance matrices $\Sigma_{\varepsilon}$ and $\Sigma_{\eta}$, respectively. ${ }^{21}$ In practice, these assumptions are not as restrictive as it might seem, because serial correlation and cross-correlation of $\eta_{t}$ and $\varepsilon_{t}$ can be introduced by including lags of these variables as elements of $X_{t}$, and redefining the disturbances in (11) and (12) to be orthogonal innovations to these processes. ${ }^{22}$ Correlation between $\eta_{t}$ and $X_{t}$ can be introduced in a similar fashion.

Policymakers minimize a quadratic loss function:

$$
\min (1-\delta) E_{t} \sum_{s=t}^{\infty} \delta^{s-t} X_{s}^{\prime} D X_{s}
$$

\footnotetext{
${ }^{20}$ It should be noted that equations in (12) that are redundant, or are not informative about $X_{t}$, in the sense that policymakers' prior variances on the corresponding elements of $Z_{t}$ are zero, should be dropped from (12). Intuitively, realizations of these components of $Z_{t}$ contain no new information, and thus are irrelevant for updating policymakers' beliefs about $X_{t}$. Mathematically, this ensures that the matrix $C \Sigma_{t-1 \mid t-1} C^{\prime}+\Sigma_{\eta}$ is nonsingular in the updating equations below.

${ }^{21}$ No difficulties arise when one allows $\Sigma_{\varepsilon}$ and $\Sigma_{\eta}$ to vary over time, so long as this variation is independent of the policy instrument.

${ }^{22}$ Aoki (1967), for example, makes this observation (pp. 38-39).
} 
where $D$ is a positive semidefinite matrix. Note that this specification does not preclude policymakers' preferences from depending on observables $Z$, since $X$ can be expanded to include elements of $Z$ as needed. Past values of $r$ can also be incorporated into $X$ and $Z$.

Policymakers choose a value for the vector of instruments $r_{t}$ at the beginning of each period $t$, conditional on all information available through the end of period $t-1$. After $r_{t}$ is chosen, the shocks $\varepsilon_{t}$ and $\eta_{t}$ are realized, and the value of the vector $Z_{t}$ is observed. Policymakers' information set at the beginning of period $t$ is thus:

$$
\mathcal{I}_{t} \equiv\left\{A, B, C, D, \delta, \Sigma_{\varepsilon}, \Sigma_{\eta}, E_{0} X_{0}, \operatorname{Var}_{0} X_{0}, Z_{s} \mid s<t\right\}
$$

where $E_{0} X_{0}$ and $\operatorname{Var}_{0} X_{0}$ denote the mean and variance of policymakers' prior (time 0 ) distribution on $X_{0}$, which is assumed to be normal.

Policymakers update beliefs about $X_{t}$ via Kalman filtering, which is the optimal inference procedure given the assumptions of normality above. Letting $\Sigma_{s \mid t}$ denote $\operatorname{Var}_{t} X_{s}$, the variance of $X_{s}$ conditional on information available at the beginning of period $t$, we have the recursive equations:

$$
\begin{aligned}
E_{t-1} X_{t-1} & =A E_{t-1} X_{t-2}+B r_{t-1} \\
E_{t-1} Z_{t-1} & =C E_{t-1} X_{t-1} \\
E_{t} X_{t-1} & =E_{t-1} X_{t-1}+\Sigma_{t-1 \mid t-1} C^{\prime}\left(C \Sigma_{t-1 \mid t-1} C^{\prime}+\Sigma_{\eta}\right)^{-1}\left(Z_{t-1}-E_{t-1} Z_{t-1}\right) \\
\Sigma_{t-1 \mid t-1} & =A \Sigma_{t-2 \mid t-1} A^{\prime}+\Sigma_{\varepsilon} \\
\Sigma_{t-1 \mid t} & =\Sigma_{t-1 \mid t-1}-\Sigma_{t-1 \mid t-1} C^{\prime}\left(C \Sigma_{t-1 \mid t-1} C^{\prime}+\Sigma_{\eta}\right)^{-1} C \Sigma_{t-1 \mid t-1}
\end{aligned}
$$

Note that the variance $\Sigma_{t \mid t}$ evolves deterministically over time, as is typical in the LQG framework. In particular, the variances of policymakers' future estimates are unaffected by their choice of the current instrument $r_{t} \cdot{ }^{23}$ This leads to separability between the estimation and control stages of policymakers' problem, and hence to the certainty equivalence result of the following proposition.

\footnotetext{
${ }^{23}$ The policymaker's choice of $r_{t}$ does not affect the signal extraction aspects of the problem-neither the variance of $\eta_{s}$ nor the variance of $X_{s}$ for any $s \geq t$-because it is assumed that the coefficient matrices $A$ and $C$ are known with certainty. This is in marked contrast to the "experimentation" motive that is present in Wieland (1998), where policymakers' choice of $r_{t}$ helps to resolve the Brainard uncertainty about the multiplicative parameters of the model.
} 
Proposition 1: The optimal solution to policymakers' problem (13), subject to the law of motion (11), observation equation (12), and information set (14) is given by:

$$
r_{t}=-\left(B^{\prime} V B\right)^{-1} B^{\prime} V A E_{t} X_{t-1}
$$

where $V$ is the "value" matrix, defined to be the unique negative semidefinite solution to the Riccati equation

$$
V=-D+\delta A^{\prime} V A-\delta A^{\prime} V B\left(B^{\prime} V B\right)^{-1} B^{\prime} V A
$$

ProOF: Bertsekas (1987), pp. 292-293.

Equation (18) is certainty-equivalent with respect to the state variable $X_{t-1}$. However, as should be clear from the previous section, certainty equivalence generally will not hold with respect to the observable variables $Z_{t-1}$. The following proposition demonstrates this fact by proving the coefficient attenuation result from the previous section for the general LQG framework. Note that by holding $\Sigma_{t-1 \mid t-1}$ fixed in what follows, the model is consistent with the interpretation that a structural break in the degree of uncertainty surrounding the indicator variables has occurred in the previous period.

Proposition 2: Suppose that the variance of the first component of $\eta_{t-1}$ in (12) is increased, in the sense that element $(1,1)$ of $\Sigma_{\eta}$ is increased while all other elements of $\Sigma_{\eta}$, and all elements of $\Sigma_{t-1 \mid t-1}$ in (15) and (16), are held fixed. Then policymakers' optimal response to observables, obtained by substituting (15) into (17), exhibits an attenuation in the response of all elements of policymakers' instrument $r_{t}$ to the first component of $Z_{t-1}$. PROOF: Recall that $r_{t}$ is a vector of instruments, hence the proposition states that the optimal setting of each of these is attenuated with respect to the first component of $Z_{t-1}$. This is intuitive because the ordering of the elements of $r_{t}$ is arbitrary.

Let $M$ denote the positive definite matrix $C \Sigma_{t-1 \mid t-1} C^{\prime}+\Sigma_{\eta}$ in equation (15), and partition $M$ into:

$$
\left[\begin{array}{ll}
M_{11} & M_{12} \\
M_{21} & M_{22}
\end{array}\right]
$$

where $M_{11}$ is a scalar, $M_{21}$ a column vector, and $M_{12}=M_{21}^{\prime}$. Letting $N$ denote $M^{-1}$ and partitioning $N$ in accordance with $M$, we have:

$$
N=\left[\begin{array}{cc}
\left|M_{22}\right| /|M| & -M_{11}^{-1} M_{12} N_{22} \\
-M_{22}^{-1} M_{21} N_{11} & M_{22}^{-1}+M_{22}^{-1} M_{21} N_{11} M_{12} M_{22}^{-1}
\end{array}\right]
$$


Let $M_{11}$ be multiplied by a factor $\lambda>1$, corresponding to the increase in $\Sigma_{\eta}$. Then $|M|$ increases because, expanding along the first row or column, $|M|=M_{11}\left|M_{22}\right|+S$, where $S$ is a sum of element-cofactor products not involving $M_{11}$, and $M_{11}$ and $\left|M_{22}\right|$ are positive. Thus, $N_{11}$ is attenuated, and it follows from (19) that $N_{21}$ is attenuated in the same proportion, say by the factor $\mu<1$. Thus the first column of $N$ is attenuated by the factor $\mu$.

Inspection of (18) reveals that $V$ is invariant to the change in $\Sigma_{\eta}$, and by the invariance of the other parameters in (15), (16), and (17), it follows that the first column of $-\left(B^{\prime} V B\right)^{-1} B^{\prime} V A \Sigma_{t-1 \mid t-1} C^{\prime}\left(C \Sigma_{t-1 \mid t-1} C^{\prime}+\Sigma_{\eta}\right)^{-1}$ is attenuated by the same factor $\mu$. These are exactly the coefficients in question, completing the proof.

One would like to be able to increase the covariances among the various components of $\eta_{t}$ as well, but unfortunately, completely general statements in this case cannot be made.

However, I may be able to say something when the variances are starting from steady state.

\section{Signal Extraction Dynamics in the Basic Model}

The analysis of the preceding sections has been essentially static in nature, in that a structural break in uncertainty was known to have occurred in the previous period. It is not clear, then, that the results still apply if the increase in uncertainty occurs several periods earlier, particularly when we take into account the fact that increased uncertainty about potential output feeds through to increased uncertainty about subsequent estimates of $X$, the unobserved state of the economy. Moreover, errors in policymakers' estimates of potential output and $X$ are serially correlated, as policymakers learn about mistakes in their estimates only slowly over time. A proper treatment of the basic model in section 2 would take these dynamic aspects of policymakers' uncertainty into consideration.

Thus, the model here will be essentially the same as in section 2. Policymakers' preferences are assumed to be of the form:

$$
\min (1-\delta) E_{t} \sum_{s=t}^{\infty} \delta^{s-t}\left(\pi_{s}^{2}+\gamma y_{s}^{2}\right)
$$


where, for ease of notation, $\pi_{s}$ now denotes the deviation of inflation from policymakers' target and $y_{s}$ denotes the output gap, both at time $s$. The economy is assumed to follow:

$$
\begin{aligned}
X_{t} & =\varphi X_{t-1}-\alpha r_{t}+\varepsilon_{t} \\
y_{t} & =X_{t}+\eta_{t} \\
\pi_{t} & =\pi_{t}^{e}+\beta X_{t}+\nu_{t}
\end{aligned}
$$

where $X_{t}$ denotes the unobserved state of the economy, $r_{t}$ the deviation of the real interest rate from its "natural" value, and $\pi_{t}^{e}$ agents' expectation of inflation, as before.

Unlike the earlier model, I allow the error term $\eta$ to be persistent:

$$
\eta_{t}=\theta \eta_{t-1}+\xi_{t}
$$

where $\theta$ is interpreted as the degree of persistence of shocks to potential output. ${ }^{24}$ The normally distributed disturbances $\varepsilon_{t}, \nu_{t}$, and $\xi_{t}$ are assumed to be orthogonal to each other, across time, and to all other variables in the system.

The timing of policymakers' observations and actions is the same as in section 2, with information set

$$
\mathcal{I}_{t} \equiv\left\{\alpha, \beta, \delta, \theta, \varphi, \sigma_{\varepsilon}^{2}, \sigma_{\nu}^{2}, \sigma_{\xi}^{2}, E_{0} X_{0}, \operatorname{Var}_{0} X_{0}, \pi_{t}^{e}, \pi_{s}^{e}, \pi_{s}, r_{s}, y_{s} \mid s<t\right\}
$$

Policymakers' optimal solution to (20), subject to (21) and (22), is given by:

$$
r_{t}=a E_{t} X_{t-1}+b E_{t} \eta_{t-1}+c \pi_{t}^{e}
$$

where $a, b$, and $c$ are constants invariant to the uncertainty surrounding $X$ and $\eta$ (see Appendix A for details). In contrast to section 2, policymakers now care about past values of $\eta$ as well as $X$. Note that because $y_{t-1}=X_{t-1}+\eta_{t-1}$, we can rewrite (23) as:

$$
r_{t}=\tilde{a} E_{t} X_{t-1}+b y_{t-1}+c \pi_{t}^{e}
$$

${ }^{24}$ This interpretation is clear if we replace (21b) with

$$
y_{t}=X_{t}+\eta_{t}+\zeta_{t}
$$

where $\eta_{t}$ represents shocks to potential output with persistence $\theta$, and $\zeta_{t}$ represents white-noise shocks to the output gap relative to the state of the economy. Clearly, the policymakers' problem in this case is essentially identical to that given in the text, so I have retained the parsimonious model there. 
where $\tilde{a} \equiv a-b$. I will refer only to the latter of the two above equations in what follows, and thus drop the tilde in (24) without risk of confusion.

As values of $y$ and $\pi$ are observed, policymakers update their beliefs about $X$ and $\eta$ by Kalman filtering. Letting $\sigma_{x_{t}}^{2}, \sigma_{\eta_{t}}^{2}$, and $\sigma_{x \eta_{t}}$ denote $\operatorname{Var}_{t} X_{t}, \operatorname{Var}_{t} \eta_{t}$, and $\operatorname{Cov}_{t}\left(X_{t}, \eta_{t}\right)$, respectively, policymakers' best estimate of $X_{t-1}$ at time $t$ is given by:

$$
\begin{aligned}
E_{t} X_{t-1}=\frac{\sigma_{\nu}^{2}\left(\sigma_{\eta_{t-1}}^{2}+\sigma_{x \eta_{t-1}}\right)}{\Delta_{t-1}} E_{t-1} X_{t-1} \\
+\frac{\sigma_{\nu}^{2}\left(\sigma_{x_{t-1}}^{2}+\sigma_{x \eta_{t-1}}\right)}{\Delta_{t-1}} y_{t-1} \\
+\frac{\beta\left(\sigma_{x_{t-1}}^{2} \sigma_{\eta_{t-1}}^{2}-\sigma_{x \eta_{t-1}}^{2}\right)}{\Delta_{t-1}}\left(\pi_{t-1}-\pi_{t-1}^{e}\right)
\end{aligned}
$$

where $\Delta_{t} \equiv \beta^{2}\left(\sigma_{x_{t}}^{2} \sigma_{\eta_{t}}^{2}-\sigma_{x \eta_{t}}^{2}\right)+\sigma_{\nu}^{2}\left(\sigma_{x_{t}}^{2}+2 \sigma_{x \eta_{t}}+\sigma_{\eta_{t}}^{2}\right)$ for all $t$. Equation (25) was derived exactly as was policymakers' estimation equation (10) in section 2 . The $E_{t-1} X_{t-1}$ term can be written as $\varphi E_{t-1} X_{t-2}-\alpha r_{t-1}$ and cascaded backward, as before.

Given an exogenous increase in uncertainty about $\eta_{s}$ at the beginning of period $s<t$, we must begin by tracing out its effects on subsequent values of $\sigma_{\eta}^{2}, \sigma_{x}^{2}$, and $\sigma_{x \eta}$. The interpretation of the exogeneity of the increase in $\sigma_{\eta_{s}}^{2}$ is that of a structural break in uncertainty surounding potential output in period $s$. The case where uncertainty about $\eta$ increases exogenously in several periods $s_{1}, s_{2}, \ldots, s_{k}$, while perhaps more interesting, is simply a positive linear combination of the effects given below, and thus does not need to be considered separately.

A straightforward computation (using equations (16) from the previous section) shows that policymakers' variances evolve according to: ${ }^{25}$

$$
\left[\begin{array}{cc}
\sigma_{x_{t+1}}^{2} & \sigma_{x \eta_{t+1}} \\
\sigma_{x \eta_{t+1}} & \sigma_{\eta_{t+1}}^{2}
\end{array}\right]=\frac{\sigma_{\nu}^{2}\left(\sigma_{x_{t}}^{2} \sigma_{\eta_{t}}^{2}-\sigma_{x \eta_{t}}^{2}\right)}{\Delta_{t}}\left[\begin{array}{rr}
\varphi^{2} & -\varphi \theta \\
-\varphi \theta & \theta^{2}
\end{array}\right]+\left[\begin{array}{cc}
\sigma_{\varepsilon}^{2} & 0 \\
0 & \sigma_{\xi}^{2}
\end{array}\right]
$$

For notational convenience, define

$$
N_{t} \equiv \frac{\sigma_{\nu}^{2}\left(\sigma_{x_{t}}^{2} \sigma_{\eta_{t}}^{2}-\sigma_{x \eta_{t}}^{2}\right)}{\Delta_{t}}
$$

\footnotetext{
${ }^{25}$ Note that $X_{t}$ and $\eta_{t}$ are correlated, since $\eta_{t-1}=y_{t-1}-X_{t-1}$. Hence $\operatorname{Cov}_{t}\left(X_{t-1}, \eta_{t-1}\right)=-\operatorname{Var}_{t} X_{t-1}$ and $\operatorname{Cov}_{t}\left(X_{t}, \eta_{t}\right)=-\varphi \theta \operatorname{Var}_{t} X_{t-1}$.
} 
which is the key term in (26). Totally differentiating $N_{t}$ with respect to $\sigma_{\eta_{s}}^{2}$, using (26), yields

$$
\frac{d N_{t}}{d \sigma_{\eta_{s}}^{2}}=\frac{\sigma_{\nu}^{4}}{\Delta_{t}^{2}}\left(\theta\left(\sigma_{x_{t}}^{2}+\sigma_{x \eta_{t}}\right)+\varphi\left(\sigma_{\eta_{t}}^{2}+\sigma_{x \eta_{t}}\right)\right)^{2} \frac{d N_{t-1}}{d \sigma_{\eta_{s}}^{2}}
$$

which is a recursive sequence terminating with:

$$
\begin{aligned}
\frac{d N_{s}}{d \sigma_{\eta_{s}}^{2}} & =\frac{\partial N_{s}}{\partial \sigma_{\eta_{s}}^{2}} \\
& =\frac{\sigma_{\nu}^{4}}{\Delta_{s}^{2}}\left(\sigma_{x_{s}}^{2}+\sigma_{x \eta_{s}}\right)^{2}
\end{aligned}
$$

The first equality in (29) follows from the assumption that the increase in uncertainty about $\eta_{s}$ in period $s$ is exogenous, while the covariance $\sigma_{x \eta_{s}}$ and variance $\sigma_{x_{s}}^{2}$, which derive from uncertainty about $\eta$ and $X$ in prior periods, are held fixed.

Note that equations (28) and (29) imply $d N_{\tau} / d \sigma_{\eta_{s}}^{2} \geq 0$ for all $\tau \geq s$. This fact, together with the relations implied by (26), helps us assess the effects of the break in uncertainty about $\eta_{s}$ on the coefficients in the period- $t$ estimation equation (25).

For example, the coefficient on the most recent inflation surprise, $\left(\pi_{t-1}-\pi_{t-1}^{e}\right)$, unambiguously increases in (25). This follows from the fact that:

$$
\frac{d}{d \sigma_{\eta_{s}}^{2}} \frac{\beta\left(\sigma_{x_{t-1}}^{2} \sigma_{\eta_{t-1}}^{2}-\sigma_{x \eta_{t-1}}^{2}\right)}{\Delta_{t-1}}=\frac{\beta}{\sigma_{\nu}^{2}} \frac{d N_{t-1}}{d \sigma_{\eta_{s}}^{2}}>0
$$

and the coefficient on the inflation surprise in (25) is positive. Thus, the finding of an amplification of the coefficient on inflation in section 2 is robust to extending the increase in uncertainty about the output gap backward any number of periods.

Similarly, the coefficient on the most recent output gap, $y_{t-1}$, is necessarily attenuated, under the assumption that $\theta>\varphi$ (which corresponds to assuming shocks to potential output are more persistent than movements in the output gap):

$$
\begin{aligned}
& \frac{d}{d \sigma_{\eta_{s}}^{2}} \frac{\sigma_{\nu}^{2}\left(\sigma_{x_{t-1}}^{2}+\sigma_{x \eta_{t-1}}\right)}{\Delta_{t-1}} \\
& \quad=\frac{\sigma_{\nu}^{2}}{\Delta_{t-1}^{2}}\left((\varphi-\theta) \sigma_{\nu}^{2}-\beta^{2}\left(\theta \sigma_{x_{t-1}}^{2}+\varphi \sigma_{x \eta_{t-1}}\right)\right)\left(\theta \sigma_{x_{t-1}}^{2}+(\theta+\varphi) \sigma_{x \eta_{t-1}}+\varphi \sigma_{\eta_{t-1}}^{2}\right) \frac{d N_{t-2}}{d \sigma_{\eta_{s}}^{2}} \\
& \quad=-\frac{\sigma_{\nu}^{2}}{\Delta_{t-1}^{2}}\left((\theta-\varphi) \sigma_{\nu}^{2}+\beta^{2} \theta \sigma_{\varepsilon}^{2}\right)\left(\theta \sigma_{\varepsilon}^{2}+\varphi \sigma_{\xi}^{2}\right) \frac{d N_{t-2}}{d \sigma_{\eta_{s}}^{2}}<0
\end{aligned}
$$


Need to show that the overall coefficient on the output gap is positive, or could assume it.

Thus, this finding from section 2 is also robust, no matter when the structural break in uncertainty occurred.

The coefficient on the lagged estimate of the state variable, $E_{t-1} X_{t-1}$, may either increase or decrease in $(25)$, according to: ${ }^{26}$

$$
\begin{aligned}
& \frac{d}{d \sigma_{\eta_{s}}^{2}} \frac{\sigma_{\nu}^{2}\left(\sigma_{\eta_{t-1}}^{2}+\sigma_{x \eta_{t-1}}\right)}{\Delta_{t-1}} \\
& \quad=\frac{\sigma_{\nu}^{2}}{\Delta_{t-1}^{2}}\left((\theta-\varphi) \sigma_{\nu}^{2}-\beta^{2}\left(\varphi \sigma_{\eta_{t-1}}^{2}+\theta \sigma_{x \eta_{t-1}}\right)\right)\left(\theta \sigma_{x_{t-1}}^{2}+(\theta+\varphi) \sigma_{x \eta_{t-1}}+\varphi \sigma_{\eta_{t-1}}^{2}\right) \frac{d N_{t-2}}{d \sigma_{\eta_{s}}^{2}} \\
& \quad=\frac{\sigma_{\nu}^{2}}{\Delta_{t-1}^{2}}\left((\theta-\varphi) \sigma_{\nu}^{2}-\beta^{2} \varphi \sigma_{\xi}^{2}\right)\left(\theta \sigma_{\varepsilon}^{2}+\varphi \sigma_{\xi}^{2}\right) \frac{d N_{t-2}}{d \sigma_{\eta_{s}}^{2}}
\end{aligned}
$$

which is positive if and only if $(\theta-\varphi)>\beta^{2} \varphi \sigma_{\xi}^{2} / \sigma_{\nu}^{2}$. Coefficients on lags of inflation and unemployment, obtained by cascading the expectational term backward in (25), may thus also go either way, depending on the sign of the derivative above. For example, if $(\theta-\varphi)>\beta^{2} \varphi \sigma_{\xi}^{2} / \sigma_{\nu}^{2}$, then the coefficient on $E_{\tau} X_{\tau}$ increases for every $\tau \geq s$. It then follows that the coefficient on the lagged inflation surprise $\left(\pi_{\tau}-\pi_{\tau}^{e}\right)$ also increases for every $\tau \geq s$ (although the change in coefficient on lags of the output gap, $y_{\tau}$, is not clear for $t-2 \geq \tau \geq s)$. If $(\theta-\varphi)<\beta^{2} \varphi \sigma_{\xi}^{2} / \sigma_{\nu}^{2}$, then the coefficient on $E_{\tau} X_{\tau}$ decreases for every $\tau \geq s$, the coefficient on $y_{\tau}$ also decreases for every $\tau \geq s$, and the change in coefficient on $\left(\pi_{\tau}-\pi_{\tau}^{e}\right)$ now becomes analytically unclear for $t-2 \geq \tau \geq s$.

I use the terminology "not clear," rather than "ambiguous," in the above because the change in coefficients may be theoretically unambiguous, but computation of the analytical derivatives and a further sign check of the result would be required to ascertain this fact. In principle, the changes in coefficients on these higher-order lags of inflation and output can be calculated analytically, but the computations quickly become very cumbersome. However, it can be shown analytically that the coefficient on the second lag of the inflation surprise, $\left(\pi_{t-2}-\pi_{t-2}^{e}\right)$, necessarily increases for $\theta>\varphi$, further corroborating the finding above that policymakers should react more aggressively to inflation.

\footnotetext{
${ }^{26}$ Note that the coefficient on $E_{t-1} X_{t-1}$ is positive, assuming again that $\theta>\varphi$.
} 
5. Conclusions 


\section{Appendix A: Solution to the Basic Model}

Here I solve the basic model of section 2 under both naive expectations $\left(\pi_{t}^{e} \equiv \pi_{t-1}\right)$ and rational expectations $\left(\pi_{t}^{e} \equiv E_{t} \pi_{t}\right)$. Recall that the model's basic equations are given by:

$$
\begin{aligned}
X_{t} & =\varphi X_{t-1}-\alpha\left(r_{t}-r^{*}\right)+\varepsilon_{t} \\
\left(y_{t}-y^{*}\right) & =X_{t}+\eta_{t} \\
\pi_{t} & =\pi_{t}^{e}+\beta X_{t}+\nu_{t}
\end{aligned}
$$

The solution under naive expectations is standard (e.g., Sargent (1987)), and is given by:

$$
r_{t}=r^{*}+a E_{t} X_{t-1}+b\left(\pi_{t-1}-\pi^{*}\right)
$$

where

$$
\left[\begin{array}{l}
a \\
b
\end{array}\right]=-\left(B^{\prime} V B\right)^{-1} B^{\prime} V A, \quad A \equiv\left[\begin{array}{cc}
\varphi & 0 \\
\beta \varphi & 1
\end{array}\right], \quad B \equiv\left[\begin{array}{c}
-\alpha \\
-\beta \alpha
\end{array}\right]
$$

and $V$ is the unique negative semidefinite solution to the Riccati equation:

$$
V=-D+\delta A^{\prime} V A-\delta A^{\prime} V B\left(B^{\prime} V B\right)^{-1} B^{\prime} V A, \quad D \equiv\left[\begin{array}{ll}
\gamma & 0 \\
0 & 1
\end{array}\right]
$$

In particular, the solution (A4) is certainty-equivalent, in that $a$ and $b$ are invariant to the stochastic parameters of the system.

For the solution under rational expectations, it is easiest to think of replacing (A3) with

$$
\pi_{t}=\lambda \pi_{t}^{e}+\beta X_{t}+\nu_{t}
$$

and consider the limit as $\lambda$ tends to one. Under both discretion and commitment, the dynamics of the problem in this case are trivial, because it has essentially no persistence (policymakers can set $X_{t}$ up to a stochastic disturbance term, and $\pi_{t}$ is a jump variable). Policymakers' problem in period $t$ thus reduces to minimizing the period- $t$ loss function $E_{t}\left[\left(y_{t}-y^{*}\right)^{2}+\gamma\left(\pi_{t}-\pi^{*}\right)^{2}\right]$.

When policymakers are short-sighted ("discretionary"), taking $\pi_{t}^{e}$ as fixed, it is easy to show that optimization and rational expectations lead to:

$$
E_{t} \pi_{t}=\frac{\beta^{2}}{\beta^{2}+\gamma(1-\lambda)} \pi^{*}, \quad E_{t}\left(y_{t}-y^{*}\right)=\frac{\beta(1-\lambda)}{\beta^{2}+\gamma(1-\lambda)} \pi^{*}
$$


which converge to $\pi^{*}$ and 0 , respectively, as $\lambda \rightarrow 1$.

Alternatively, when policymakers are far-sighted ("committed"), optimization and rational expectations lead to:

$$
E_{t} \pi_{t}=\frac{\beta^{2}}{\beta^{2}+\gamma(1-\lambda)^{2}} \pi^{*}, \quad E_{t}\left(y_{t}-y^{*}\right)=\frac{\beta(1-\lambda)}{\beta^{2}+\gamma(1-\lambda)^{2}} \pi^{*}
$$

which likewise converge to $\pi^{*}$ and 0 as $\lambda \rightarrow 1$ (not surprising, since setting $\pi_{t}=\pi^{*}$ and $\left(y_{t}-y^{*}\right)=0$ in expectation is the global optimum, and this was achieved even under discretion).

Thus, under rational expectations, we can regard policymakers as solving the following more standard linear-quadratic problem:

$$
\begin{aligned}
X_{t} & =\varphi X_{t-1}-\alpha\left(r_{t}-r^{*}\right)+\varepsilon_{t} \\
\left(y_{t}-y^{*}\right) & =X_{t}+\eta_{t} \\
\pi_{t} & =\pi^{*}+\beta X_{t}+\nu_{t}
\end{aligned}
$$

for which the optimal solution is:

$$
r_{t}=r^{*}+a E_{t} X_{t-1}+b\left(\pi^{*}-\pi^{*}\right)
$$

where $a$ and $b$ are given by equation (A5), exactly as before. Obviously, the $\left(\pi^{*}-\pi^{*}\right)$ term can be dropped, but leaving it facilitates comparison to (A5) and the single solution given in the main body of the text,

$$
r_{t}=r^{*}+a E_{t} X_{t-1}+b\left(\pi_{t}^{e}-\pi^{*}\right)
$$

It is then not hard to solve explicitly for $V$ in (A6) and show that $a$ and $b$ are necessarily positive.

Need to redo whole section using slightly more general model of section 4 . 


\section{References}

Aoki, Kosuke. "On the Optimal Monetary Policy Response to Noisy Indicators," unpublished manuscript (1999), Princeton University.

Aoki, Masanao. Optimization of Stochastic Systems: Topics in Discrete-Time Systems (Academic Press: New York, 1967).

Bertsekas, Dimitri. Dynamic Programming (Prentice-Hall: Englewood Cliffs, NJ, 1987).

Brainard, William. "Uncertainty and the Effectiveness of Policy," AEA Papers \& Proceedings 57 (1967), pp. 411-425.

Clarida, Richard, Jordi Galí, and Mark Gertler. "Monetary Policy Rules in Practice: Some International Evidence," European Economic Review 42, pp. 1033-1068.

Drew, Aaron and Benjamin Hunt. "Efficient Simple Policy Rules and the Implications of Potential Output Uncertainty," Reserve Bank of New Zealand Discussion Paper G99/5 (1999).

Estrella, Arturo and Frederic Mishkin. "Rethinking the Role of NAIRU in Monetary Policy: Implications of Model Formulation and Uncertainty," in Monetary Policy Rules, ed. John Taylor (1999), pp. 405-430.

Mankiw, N. Gregory and Matthew Shapiro. "News or Noise: An Analysis of GNP Revisions," Survey of Current Business (1986), pp. 20-25.

McNees, Stephen. "An Assessment of the 'Official' Economic Forecasts," New England Economic Review (1995), pp. 13-23.

Onatski, Alexei and James Stock. "Robust Monetary Policy Under Model Uncertainty in a Small Model of the U. S. Economy," unpublished manuscript (1999), Harvard University.

Orphanides, Athanasios. "The Quest for Prosperity Without Inflation," unpublished manuscript (1999), Federal Reserve Board.

Orphanides, Athanasios. "Monetary Policy Evaluation with Noisy Information," Federal Reserve Board Finance and Economics Discussion Series 1998-50 (1998).

Orphanides, Athanasios, Richard Porter, David Reifschneider, Robert Tetlow, and FREDERICO FinAN. "Errors in the Measurement of the Output Gap and the Design of Monetary Policy," Federal Reserve Board Finance and Economics Discussion Series 1999-45 (1999).

Romer, Christina And DAvid Romer. "Federal Reserve Private Information and the Behavior of Interest Rates," NBER Working Paper 5692 (1996); a more recent version is forthcoming, A.E.R. (2000).

Rudebusch, Glenn. "Is the Fed Too Timid? Monetary Policy in an Uncertain World," Federal Reserve Bank of San Francisco Working Paper 99-05 (1999a).

Rudebusch, Glenn. "Assessing Nominal Income Rules for Monetary Policy with Model and Data Uncertainty," unpublished manuscript (1999b), European Central Bank Conference.

SACK, Brian. "Does the Fed Act Gradually? A VAR Analysis," Federal Reserve Board Finance and Economics Discussion Series 1998-17 (1998).

Sargent, Thomas. Dynamic Macroeconomic Theory (Harvard University Press: Cambridge, MA, 1987). 
Smets, Frank. "Output Gap Uncertainty: Does it Matter for the Taylor Rule?" unpublished manuscript (1998), European Central Bank.

Svensson, Lars And Michael Woodford. "Indicator Variables for Optimal Policy," unpublished manuscript (1999), European Central Bank Conference.

Taylor, John. "Discretion versus Policy Rules in Practice," Carnegie-Rochester Conference Series on Public Policy 39 (1993), pp. 195-214.

WiEland, Volker. "Monetary Policy and Uncertainty About the Natural Unemployment Rate," Federal Reserve Board Finance and Economics Discussion Series 1998-22 (1998). 